\title{
2. Komplementärmedizin in der Gynäkologie
}

\author{
HANS-WOLFGANG HOEFERT
}

\section{Einleitung}

Komplementär-alternative Heilmethoden erfreuen sich in der Gynäkologie relativ großer Beliebtheit unter den Patientinnen. Im Folgenden wird dargestellt, wie sich aus Sicht der evidenzbasierten Medizin solche Methoden in verschiedenen Indikationsbereichen bewährt haben. Zunächst wird ein kurzer Überblick gegeben über die wesentlichen Nutzungsmotive und Nutzercharakteristika. Der erste Abschnitt konzentriert sich auf die Anwendung von Komplementärmedizin (KM) zur Steigerung oder Wiederherstellung von Sexualität und Fertilität, der zweite Abschnitt auf die Anwendung in der Schwangerschaft und rund um die Geburt, der dritte Abschnitt auf die Anwendung während der Menopause. Daran schließen sich zwei weitere Abschnitte an, die sich auf die Anwendung bei Ovarialoder Brustkrebs bzw. bei einzelnen Indikationen wie dem prämenstruellen Syndrom, der Dysmenorrhoe, der Endometriose oder der Vaginitis beziehen. Der Beitrag wird abgeschlossen mit einer zusammenfassenden Würdigung der Anwendungsmöglichkeiten von KM-Methoden.

\subsection{Epidemiologie von gynäkologischen Störungen und Inanspruchnahme von KM}

Die Prävalenz gynäkologischer Erkrankungen und Beschwerden lässt sich weniger gut bestimmen als in anderen medizinischen Fachdisziplinen. Geht man aus von den Krankschreibungsursachen, dann stehen Entzündungen im Ovarial- und 
Scheidenbereich, Unterbauchschmerzen und Störungen der Regelblutung im Mittelpunkt. In der gynäkologischen Arztpraxis ist das Behandlungsspektrum wesentlich breiter; es reicht von objektivierbaren körperlichen Manifestationen (z.B. Entzündungen, Krebserkrankungen) bis hin zu eher psychischen Symptomatiken, die sich durch natürliche Prozesse wie Menstruation und Schwangerschaft, aber auch durch Probleme mit der eigenen Frauenrolle in den verschiedenen Lebensphasen sowie wie der Partnerschaft ergeben ${ }^{10}$.

Die Tatsache, dass komplementär-alternative Heilmethoden ohnehin stärker von Frauen als von Männern genutzt werden, spiegelt sich auch in der Gynäkologie wider, wenngleich die Präferenzen für bestimmte Methoden im internationalen Vergleich unterschiedlich gewichtet sind. Dieser Vergleich lässt erkennen, dass die stärkste Nutzung in verschiedenen Ländern bei den Frauen im mittleren Alter liegt, wobei in Deutschland an der Spitze Verfahren wie Akupunktur, Homöopathie und Aromatherapie präferiert werden (Münstedt et al. 20o9a). In den USA rangieren an der Spitze biologische Therapien, gefolgt von sog. Mind-BodyTherapien (Upchurch et al. 2007), in Australien Massage-Therapien, Kräutertherapien, Entspannungstechniken, Nahrungsmittelzusätze, Aromatherapie, Homöopathie und Akupunktur (Hall et al. 2012), und in Kanada bei Frauen in der Menopause Vitamine, Entspannungstechniken, Yoga und Meditation, SojaProdukte und Beten (Lunny \& Fraser 2010). Die jeweilige Therapie-Präferenz wird mitbestimmt durch den kulturellen Hintergrund (z.B. stärkere Präferenz für das Beten in den USA und Kanada als in Europa), die finanziellen Mittel, die Zugänglichkeit zu KM-Spezialisten, welche in Städten größer ist als auf dem Lande, und schließlich auch durch die Art der Methode (z.B., ob die Methode eine körperliche Berührung vorsieht oder nicht, vgl. Lindenmeyer et al. 2011).

Fragt man nach den Nutzungsmotiven, so werden neben den Überzeugungen, KM-Methoden seien sicherer (mit weniger Nebenwirkungen als herkömmliche Medikamente), auch Überzeugungen genannt, sie seien „philosophisch kongruenter“ und würden die weibliche Autonomie unterstützen (Hall et al. 2012) oder würden sich im Einklang befinden mit bestimmten politisch-kritischen oder feministischen Positionen (Lindenmeyer et al. 2011). Allerdings werden KMMethoden nur relativ selten ausschließlich und anstelle konventioneller Verfahren verwendet, meist kommen beide Methodentypen parallel zu Anwendung, wovon der behandelnde (konventionelle) Gynäkologe allerdings oft nichts weiß: In einer repräsentativen US-amerikanischen Untersuchung zum KM-Gebrauch nutzten zwar 36\% der Frauen KM-Methoden, wovon aber nur die Hälfte ihrem Gynäkologen davon berichtete (Harrigan 2011). Die Bereitschaft, darüber Auskunft zu geben, scheint in Privatpraxen größer zu sein als im klinischen Kontext (Smith et al. 2012).

Aber auch der Parallelgebrauch bleibt offenbar nicht ohne Auswirkung auf das Präventionsverhalten: So nehmen beispielsweise KM-Nutzerinnen weniger an

\footnotetext{
${ }^{10} \mathrm{Vgl}$. www.frauenaerzte-im-netz.de
} 
Chlamydien-Tests oder, spezieller, Nutzerinnen der Naturheilkunde weniger an Mammografien teil, während der sog. Pap-Test von allen Frauen gleichmäßig in Anspruch genommen wird. Die Teilnahme an Krebsvorsorge-Tests scheint bei Frauen mit einem komplementären Nutzungsverhalten größer zu sein als bei einem alternativen Verhalten (Downey et al. 2009).

Ärzte sind nur zum Teil überzeugt von der Wirksamkeit von KM-Methoden (vgl. Münstedt et al. 2009) bzw. bevorzugen selbst bei einer generell positiven Einstellung zur KM zum Teil andere Methoden als ihre Patientinnen (Furlow et al. 2008). Dabei scheinen gynäkologische Onkologen und weibliche Ärzte eher zu einer KM-Empfehlung bereit zu sein als andere Onkologen und männliche Ärzte (Rhode et al. 2008). Wieweit bei solchen Präferenzen die wissenschaftliche Evidenzbasierung eine Rolle spielt, nach der die Wirksamkeit von CAM im gynäkologischen Bereich nicht allzu überzeugend ist (Ernst \& Watson 2011), oder nicht vielmehr persönliche Überzeugungen wirksam sind, die Teil des individuellen Weltbildes sind, ist eine offene Frage. Außerdem ist zu berücksichtigen, dass nicht immer die Überzeugungen und Ratschläge von Ärzten für die Patientenentscheidungen zugunsten von KM entscheidend sind, sondern häufig diejenigen aus dem sozialen Umfeld oder von Hebammen (vgl. Münstedt et al. 2009).

\subsection{Sexualität und Fertilität}

Die Literaturlage zur Verwendung von KM-Therapien im Hinblick auf eine Verbesserung von Sexualfunktionen und sexueller Zufriedenheit ist weitgehend spekulativ. So wird beispielsweise angenommen, dass speziell „östliche“ Techniken wie Yoga, Akupunktur oder Achtsamkeitsmeditation dazu beitragen können, das Bewusstsein für die eigene Sexualität zu schärfen (vgl. Brotto et al. 2008, speziell für Yoga Brotto et al. 2009, Dhikav et al. 2010), wobei entsprechende Nachweise noch weitgehend ausstehen. Allerdings scheint insbesondere Achtsamkeitsmeditation zu einer besseren Wahrnehmung der eigenen Körperfunktionen beizutragen (Silverstein et al. 2011). Dagegen kann die Verwendung von Pflanzen zur Behebung einer „sexuellen Dysfunktion“ aufgrund der wenigen Studien kaum empfohlen werden (Mazaro-Costa et al. 2010).

Relativ wenig ist auch bekannt über die Nutzung von KM-Therapien zur Vergrößerung der Fertilitätschancen oder der jeweiligen Nutzungsmotive (vgl. Rayner et al. 2011). Einige Befunde deuten darauf hin, dass KM-Therapien nur von einer Minderheit unfruchtbarer Paare bzw. eher von wohlhabenderen Paaren, zumal solchen, die von der Wirksamkeit von KM überzeugt sind, genutzt werden (Smith et al. 2010). Am populärsten scheint dabei die Verwendung von Akupunktur zu sein (vgl. Weiss et al. 2011), die durch einen Anstieg der Geburtsrate gestützt wird (Cheong et al. 2008), sich offenbar aber weniger eignet für die Behebung sexueller Dysfunktionen (Ernst et al. 2011) und auch bei der In-vitro-Fertilisation ohne Wirkung bleibt (El-Toukhy et al. 20o8, Madaschi et al. 2010). Bei der letztgenannten Störung scheint die Verwendung von chinesischen Phytopharmaka erfolgver- 
sprechender (Ried \& Stuart 2011). Die wesentlichen Motive für die KM-Nutzung bei Infertilität liegen einer kanadischen Untersuchung zufolge (Schaffir et al. 2009) in dem Fehlen von Nebenwirkungen und darin, dass damit eine konventionelle Therapie unterstützt werden könne.

Als wichtigste Methoden wurden in der Untersuchung von Schaffir et al. (2009) Beten, Änderung der Sexualpraktiken und Änderung der Ernährungsgewohnheiten genannt. In einem US-amerikanischen Review (Clark et al. 2013a) hatten sich vor allem Akupunktur, Selenium-Zusätze, Gewichtsabnahme und psychotherapeutische Interventionen bewährt, um die Fertilität zu erhöhen. Anzumerken ist, dass nur etwa ein Drittel der Patientinnen ihrem behandelnden Arzt über den Gebrauch von KM berichtet, wobei der Hauptgrund schlicht darin besteht, dass sie nicht danach gefragt werden (Clark et al. 2013b).

Interessant ist diesbezüglich der Befund von Boivin \& Schmidt (2009), dem zufolge die Geburtswahrscheinlichkeit durch KM-Gebrauch absinkt, was zum einen darauf zurückgeführt werden könne, dass die Frauen bereits viele erfolglose Versuche hinter sich haben, sich ihre positiven Erwartungen verringern und sie entsprechend unter Stress stehen, der sich negativ auf die Empfängnis auswirkt, zum anderen darauf, dass parallel auch konventionelle Verfahren und vor allem mehr als eine KM-Methode erprobt wird, sodass sich mögliche positive Effekte (wie bei einer Monotherapie von Akupunktur) gegenseitig aufheben.

\subsection{Schwangerschaft und Geburt}

Viele Frauen glauben, den Verlauf der Schwangerschaft und vor allem die Chancen für eine zeitgerechte und komplikationslose Geburt durch die Anwendung von bestimmten KM-Methoden wie Yoga, Entspannungstechniken oder Akupunktur bzw. durch die Einnahme von Vitaminen und Nahrungsergänzungsmitteln unterstützen zu können. In Deutschland werden speziell Homöopathie, Akupunktur und Phytotherapie bevorzugt (Kalder et al. 2011). Die entsprechenden Prävalenz-Schätzungen reichen von $1 \%$ bis $87 \%$, wobei die Schätzunsicherheit hauptsächlich darauf zurückzuführen ist, dass die Frauen ihrem Arzt gegenüber davon nicht berichten (Hall et al. 2011). Das Anwendungsspektrum von KMMethoden ist dabei recht breit, es betrifft Übelkeit vor allem in der ersten Schwangerschaftsphase, Rückenbeschwerden ebenso wie depressive Zustände (vgl. Field 2008).

Die Wahl von KM-Methoden entspricht den Bedürfnissen nach Risikominimierung und Autonomie bei der Gestaltung der Schwangerschaft (im Überblick Adams et al. 2009, Louik et al. 2010, Mitchell 2010). Ein weiteres Motiv scheint darin $\mathrm{zu}$ bestehen, dass viele KM-Nutzerinnen glauben, mit KM eine bessere Kontrolle über den Verlauf der Schwangerschaft zu behalten und etwas zu tun, was ihrem Weltbild entspricht, wobei sie über dieses Weltbild zumeist nicht mit ihrem Arzt sprechen (Warriner et al. 2013). 
Im Einzelnen scheinen bestimmte Mind-Body-Methoden wie Autogenes Training, Biofeedback, Hypnose, Meditation, Tai-Chi oder Yoga einen positiven Effekt auf die mit einer Schwangerschaft oft einhergehenden Ängste zu haben (Marc et al. 2011). Übelkeit während der Schwangerschaft lässt sich offenbar durch Ingwer-Produkte und Vitamin B6 mildern, während der Effekt von Verfahren wie Akupunktur und Akupressur eher unklar ist (Matthews et al. 2010, Smith \& Cochrane 2009). Ob und wie häufig Akupunktur verwendet wird, dürfte auch von dem jeweiligen Krankenhaus abhängen, in dem eine Patientin ihre Entbindung vorbereitet, sowie von ihrem Bildungsstatus (Zuwachs an KM-Gebrauch mit dessen Höhe), wobei der KM-Gebrauch auch mit einer häufigeren Verwendung von Analgetika einhergeht (Münstedt et al. 2011).

Schmerzen im Becken- und Lendenbereich insbesondere in der Spätphase der Schwangerschaft lassen sich osteopathisch gut behandeln; der Effekt anderer körperlicher Übungen ist eher gering (Pennick \& Young 2007). Aber auch bei entzündlichen Prozessen im Beckenbereich werden nach einer US-amerikanischen Studie (Slavin et al. 2010) hauptsächlich Vitamine, Phytopharmaka und Akupunktur verwendet, wenn man von dem hierzulande weniger üblichen Beten absieht.

Nicht zu vergessen ist schließlich, dass bestimmte Methoden (z.B. Yoga-Programme, vgl. Curtis et al. 2012) geeignet sind, das Selbstvertrauen in die Fähigkeit zur Bewältigung von Schmerzen und Beschwerden zu stärken und auf diese Weise medikamentöse Interventionen überflüssig machen.

Abgesehen von solchen Methoden, die direkt oder indirekt (über die Psyche) auf eine Entspannung der Muskulatur abzielen, sind bei vielen Frauen Vitamine, Nahrungsmittelzusätze und Kräuter beliebt, von denen sie sich eine direkte Wirkung auf ihr Wohlbefinden und die Chancen einer gesunden Geburt erhoffen. Die folgende Tabelle 8 zeigt auf der Grundlage von neueren Reviews, dass die Evidenz für eine Effektivität solcher Mittel eher gering ist.

Tab. 8 KM-Interventionen während der Schwangerschaft

\begin{tabular}{|c|c|c|}
\hline Mittel & Ergebnis in Kurzform & Quelle \\
\hline $\begin{array}{l}\text { Vitamine allge- } \\
\text { mein }\end{array}$ & verhindern keine Früh- oder Fehlgeburt & Rumbold et al. 2011 \\
\hline Vitamin B6 & $\begin{array}{l}\text { Evidenz unzureichend, lediglich Verbesserung des } \\
\text { Zahnzustandes }\end{array}$ & Thaver et al. 2006 \\
\hline Vitamin D & $\begin{array}{l}\text { erhöht Serum Vitamin D-Konzentration, dadurch poten- } \\
\text { ziell positiver Effekt }\end{array}$ & De-Regil et al. 2012 \\
\hline Vitamin $\mathrm{C}$ und $\mathrm{E}$ & verhindern keine Komplikationen bei der Geburt & Rumbold et al. 2006 \\
\hline Folate & $\begin{array}{l}\text { verhindern Defekte der neuralen Tuben, sonst keine } \\
\text { weiteren geburtlichen Defekte }\end{array}$ & De-Regil et al. 2010 \\
\hline
\end{tabular}




\begin{tabular}{|c|c|c|}
\hline Mittel & Ergebnis in Kurzform & Quelle \\
\hline Eisen & $\begin{array}{l}\text { erhöht das Risiko eines Hämoglobinnstiegs, wobei } \\
\text { gelegentliche und nicht tägliche Einnahme ausreicht }\end{array}$ & Pena-Rosas et al. 2012 \\
\hline Zink & kein Effekt bei Frauen mit normalem Ernährungsstatus & Mori et al. 2012 \\
\hline $\begin{array}{l}\text { Mikro- } \\
\text { Nutrientien }\end{array}$ & $\begin{array}{l}\text { verringern das Risiko eines zu niedrigen Geburtsge- } \\
\text { wichts }\end{array}$ & Haider \& Bhutta 2012 \\
\hline Chin. Kräuter & $\begin{array}{l}\text { keine Aussage möglich, ob sie eine Bauchhöhlen- } \\
\text { schwangerschaft begünstigen oder nicht }\end{array}$ & Qu et al. 2011 \\
\hline Chin. Kräuter & $\begin{array}{l}\text { unzureichende Evidenz, ob damit einer Geburtskompli- } \\
\text { kation vorgebeugt werden kann }\end{array}$ & Li et al. 2012 \\
\hline $\begin{array}{l}\text { Kräuter allge- } \\
\text { mein }\end{array}$ & $\begin{array}{l}\text { vergrößern nicht das Risiko eines zu geringen Geburts- } \\
\text { gewichts }\end{array}$ & Moussaly \& Berard 2012 \\
\hline
\end{tabular}

Im Übrigen versuchen Frauen, bestimmte Reizstoffe zu vermeiden, mit denen ihre Schwangerschaft gefährdet werden könnte. Solche Sorgen (einmal abgesehen von Rauchen und Alkohol) scheinen jedoch unbegründet zu sein, da weder Koffein (Jahanfar \& Jaafar 2012) noch Salz (Duley \& Henderson-Smart 2009) einen nennenswerten Effekt haben.

KM-Methoden werden auch bei der Geburt eingesetzt. Inwieweit das jeweilige Ambiente dabei unterstützend wirken kann, ist eine noch offene Frage, da sich speziell das von KM-Anhängerinnen befürwortete Ambiente sehr stark unterscheiden; spezielle Geburtszentren in Krankenhäusern scheinen immer weniger medizinische Interventionen erforderlich zu machen (Hodnett et al. 2012). Die folgende Tabelle 9 gibt einen Überblick über die Effektivität verschiedener KMInterventionen, speziell zur Reduzierung des Geburtsschmerzes.

Tab. 9 KM-Interventionen bei der Geburt

\begin{tabular}{|c|c|c|}
\hline Methode & Ergebnis in Kurzform & Quelle \\
\hline \multirow[t]{4}{*}{ Akupunktur } & trägt zu einer größeren Zufriedenheit mit dem & Smith et al. 2011a \\
\hline & Schmerzmanagement bei und hilft bei der Reduk- & Lim et al. 2009 \\
\hline & tion pharmakologischer Interventionen & Tournaire \& Theau-Yonneau \\
\hline & & 2007 \\
\hline Akupressur/Eismassage & $\begin{array}{l}\text { tragen beide zur Schmerzreduktion bei, der Effekt } \\
\text { der Eismassage halt länger an }\end{array}$ & Hajiamini et al. 2012 \\
\hline Aromatherapie & zu wenige Studien für eine Aussage & Smith et al. 2011b \\
\hline Biofeedback & unzureichende Evidenz & Barragan et al. 2011 \\
\hline Entspannungstechniken & $\begin{array}{l}\text { und Yoga können Schmerz reduzieren, während } \\
\text { für Musik und Audio-Analgesie die Evidenz unzu- }\end{array}$ & Smith et al. 2011c \\
\hline
\end{tabular}




\begin{tabular}{|c|c|c|}
\hline Methode & Ergebnis in Kurzform & Quelle \\
\hline \multirow[b]{3}{*}{ Homöopathie } & \multicolumn{2}{|l|}{ reichend ist } \\
\hline & $\begin{array}{l}\text { tragen generell zur Reduzierung von Stress und } \\
\text { Angst bei, haben aber keinen Effekt auf den } \\
\text { Geburtstermin }\end{array}$ & Khianman et al. 2012 \\
\hline & unzureichende Evidenz & Smith 2010 \\
\hline Hypnose & $\begin{array}{l}\text { unzureichende Evidenz (Sado), in kleineren } \\
\text { Studien Reduzierung der Schmerzintensität und } \\
\text { Verkürzung der Geburtsdauer (Madden) }\end{array}$ & $\begin{array}{l}\text { Sado et al. } 2012 \\
\text { Madden et al. } 2012\end{array}$ \\
\hline Magnesium & $\begin{array}{l}\text { Evidenz für eine Stabilisierung bei drohender } \\
\text { Frühgeburt (Doyle), keine Evidenz (Han) }\end{array}$ & $\begin{array}{l}\text { Doyle et al. } 2009 \\
\text { Han et al. } 2010\end{array}$ \\
\hline Massage & $\begin{array}{l}\text { Massage und Reflexologie lindern die Schmerz- } \\
\text { empfindung }\end{array}$ & Smith et al. 2012 \\
\hline Nahrungsmittelzusätze & unzureichende Evidenz & Say et al. 2010 \\
\hline Probiotika & $\begin{array}{l}\text { zu wenige Studien für eine Aussage, aber Redu- } \\
\text { zierung des vaginalen Bakterienbefalls }\end{array}$ & Othman et al. 2012 \\
\hline TENS & $\begin{array}{l}\text { tendenziell Schmerzreduzierung, aber keine } \\
\text { konsistenten Ergebnisse }\end{array}$ & Dowswell 2011 \\
\hline Yoga & $\begin{array}{l}\text { wirkt stressreduzierend, erhöht Lebensqualität } \\
\text { während der Schwangerschaft }\end{array}$ & Curtis et al. 2012 \\
\hline
\end{tabular}

Anzumerken ist, dass das Interesse von Frauen an KM-Methoden mit sich näherndem Geburtstermin ansteigt, dass aber dann, wenn eine Frühgeburt eintritt, das Interesse an KM sinkt, was möglicherweise mit unzureichender Aufklärung bzw. ungerechtfertigten Erwartungen während der Schwangerschaft zusammenhängt (Münstedt et al. 2013).

Speziell für die Einleitung einer Geburt schlagen Hall et al. (2011) neben Bruststimulation Akupunktur und Himbeerblätter vor, warnen vor dem Gebrauch von Rizinusöl, Schlüsselblumen-Extrakt oder Frauenwurz, weil diese zu Komplikationen beitragen können, und bescheinigen der Homöopathie mangelnde Evidenz. Verschiedene Autoren weisen jedoch darauf hin, dass man Frauen, die von der Wirksamkeit einer bestimmten Methode überzeugt sind (z.B. auch Homöopathie) diese nicht verweigern sollte (so auch Hall et al. 2011).

\subsection{Menopause}

Typische menopausale Symptome bestehen in Hitzewallungen, Schwitzanfällen, vaginaler Trockenheit und Schlafstörungen. Insbesondere die vasomotorischen Symptome (Schwitzanfälle u.ä.) sind Gegenstand sowohl der Hormonersatzthe- 
rapie als auch der KM. Nicht-hormonelle Therapien bzw. KM-Therapien werden zumeist wegen der relativ geringen Nebenwirkungen oder wegen eines bei längerem hormonellen Gebrauch bestehenden Brustkrebs-Risikos bevorzugt. Daneben behelfen sich viele Frauen mit Verhaltensmodifikationen wie Erhaltung einer niedrigen Körpertemperatur, Vermeidung scharfer Speisen oder Tragen lockerer Kleidung, ohne auf eine spezielle Therapie zurückzugreifen (vgl. Morrow et al. 2011). In einer neueren deutschen Befragung von schwangeren Frauen (von Studnitz et al. 2013) zeigte sich, dass die meisten Frauen die Veränderung ihres Lebensstils (Ess- und Schlafgewohnheiten) für am wirksamsten im Umgang mit menopausalen Symptomen hielten. Für wirksam hielten sie außerdem Johanniskraut und Traubensilberkerze, nicht für wirksam dagegen Hormon-Yoga, Akupunktur und Homöopathie. Einer US-amerikanischen Metaanalyse zufolge (Shams et al. 2010) werden sehr häufig Präparate mit schwarzem Frauenfuß (black cocosh) verwendet; von einem Viertel der Frauen wird diese Präparate als wirksam bewertet. In einer deutschen Studie (Laakmann et al. 2012) zeigte sich, dass oft erst die Kombination verschiedener Kräuter (und nicht einzelne Kräuter für sich) zu einer Verbesserung klimakterischer Beschwerden beiträgt.

Speziell zum Problem der Hitzewallungen in der Menopause liegt ein neueres Cochrane-Review vor (Dodin et al. 2013), nach dem bei der Verwendung von Akupunktur diese Symptome zwar nicht seltener auftreten, aber als weniger schwer erlebt werden. Akupunktur rangierte in ihrer Wirksamkeit bei Hitzewallungen zwar hinter einer Hormontherapie, war allerdings wirksamer als gar keine Therapie.

Geht man aus von neueren Reviews zur Effektivität von KM-Therapien, dann ergibt sich ein differenziertes Bild: So scheinen sog. Mind-Body-Therapien (Yoga, Meditation, Tai Chi, Entspannungstechniken u.ä.) vor allem einen unspezifischen Nutzen für das allgemeine Wohlbefinden zu bringen, weniger dagegen für eine Milderung der o.e. vasomotorischen Symptome (vgl. Innes et al. 2010, Pitkin 2012, Thacker 2011, Wong et al. 2009). Der spezifische Nutzen von KM-Therapien einschließlich der Mind-Body-Therapien, pflanzlicher Therapien sowie Verfahren wie Akupunktur oder Homöopathie wird hier von einigen Review-Autoren generell als nicht nachweisbar bis gering eingeschätzt (vgl. Nedrow et al. 20o6, Pachman et al. 2010). Dabei ist zu bedenken, dass das subjektive Leiden an diesen Symptomen interindividuell recht stark variiert: Als „schwer“ werden einer älteren Studie von Keenan et al. (2003) zufolge die Symptome eher von Frauen bezeichnet, welche sowohl konventionelle als auch KM-Therapien in Anspruch nehmen, als von Frauen, die entweder nur die eine oder andere Therapieart oder gar keine Therapien nutzt.

Etwas differenzierter kennzeichnet Pitkin (2012) die Evidenznachweise für Akupunktur als widersprüchlich, Nahrungszusätze als wirkungslos, unter den Phytopharmaka das bei Depressionen bewährte Johanniskraut als bestenfalls indirekt wirksam, Traubensilberkerze (Cimifuga racemosa) und Cingko biloba als zumindest in einigen Studien wirksam, Ginseng, Dong Quai als nicht wirksam. Chinesische Kräu- 
termedizin hat eine Reihe von positiven Effekten auf die menopausale Symptomatik, jedoch ohne die Nebenwirkungen einer vergleichbaren Hormontherapie (im Überblick Xu et al. 2012).

Unter den Phytoöstrogenen haben sich Soja-Zusätze in einigen Studien bewährt, während Rotklee sich in recht vielen Studien bewährt hat. In ihrem schon etwas älteren Review hatten Huntley \& Ernst (2003) bereits dem Rotklee gute Bewährungschancen bescheinigt (anderen Kräutern hingegen keine überzeugende Evidenz), die bisher aber noch nicht nachgewiesen werden konnten (vgl. Borelli \& Ernst 2008, Leach \& Moore 2012). Der eindeutige Nachweis einer spezifischen Wirkung durch Phytoöstrogene steht nach wie vor aus (vgl. Lethaby et al. 2007), obwohl einige Studien hier inzwischen durchaus zu positiven Aussagen gelangen (z.B. Sunita \& Pattanayak 2011).

Körperliche Bewegungsübungen scheinen keinen spezifischen Effekt zu haben, obwohl sie einen kleinen Nutzen bringen im Vergleich zur Nicht-Bewegung (Daley et al. 2011). Die Evidenz für die Wirkung von Yoga ist bestenfalls moderat für kurzfristige Effekte bei psychologischen Symptomen, gering hingegen bei somatischen, vasomotorischen und urogenitalen Symptomen (Cramer et al. 2012).

Entgegen einer weit verbreiteten Meinung hat sich Akupunktur hier diskutierten Indikation offenbar nicht bewährt (Cho et al. 2009, Sideras et al. 2010, Vincent et al. 2007) und die Evidenz von Yoga ist nicht überzeugend (Lee et al. 2009). Angesichts des Risikos langfristiger hormoneller Therapien scheinen damit zumindest einige KM-Verfahren die Methoden der Wahl bei der Milderung vasomotorischer Symptome zu sein, wenngleich ihre Wirkung nicht an diejenige der medikamentösen Verfahren heranreicht (vgl. Cheema et al. 2007, Hall et al. 2011, Shou et al. 2011). Entsprechend empfiehlt die Nordamerikanische Menopause-Gesellschaft bei milden Symptomen zunächst Lebensstil-Veränderungen, eventuell in Kombination mit Isoflavonen, Rotklee oder Vitamin E, bei mittleren bis schweren Symptomen jedoch den Gebrauch Östrogen-haltiger Produkte (NAMS 2004).

\subsection{Krebserkrankungen}

Mehr als die Hälfte aller von Brust- oder Ovarialkrebs betroffenen Frauen erprobt irgendeine KM-Methode. Die entsprechenden Informationen und Ratschläge beziehen sie nicht in erster Linie von ihrem behandelnden Gynäkologen, sondern aus dem Internet, aus den Medien und aus dem Bekanntenkreis. Die Hauptgründe für den Gebrauch sind, die allgemeine Lebensqualität zu verbessern, die Nebenwirkungen einer Chemo- oder Radiotherapie zu mildern, und erst an dritter Stelle, den Krebs selbst zu bekämpfen (vgl. Navo et al. 2004). Auch in einer deutschen Untersuchung war der Hauptgrund für die KM-Nutzung der bessere Umgang mit Therapie-bedingten Beschwerden (Fasching et al. 2007).

KM-Nutzerinnen haben zumeist - wie KM-Nutzer generell - einen höheren Bildungsgrad, ein höheres Alter und zeichnen sich nicht durch eine erhöhte 
Rezidiv-Besorgnis aus (Mueller et al. 2008). Sie berichten über vielfältigere körperliche und psychische Beschwerden als Nicht-Nutzerinnen (Saquib et al. 2011). Eine entsprechende häufigere Komorbidität mit nicht Krebs-bedingten $\mathrm{Be}$ schwerden zeigt sich auch bei Langzeit-Überlebenden im Abstand von etwa 10 Jahren nach der Erstdiagnose, insbesondere bezüglich der psychischen Funktionalität (Carpenter et al. 2009).

Wenn KM-Methoden aus allgemeineren Gründen (Verbesserung der Lebensqualität) genutzt werden, dann kommen vor allem Methoden wie Meditation und spirituelle Techniken infrage (Saquib et al. 2011), bei körperlichen Beschwerden eher biologische Verfahren wie z.B. Soja-Nahrungsergänzungsmittel, Flachsöl, Schneckenklee (Alfalfa), oder Rotklee (Ma et al. 2011, Roberts 2010). Frauen, die Hypnose erproben, berichten nicht nur über weniger Hitzewallungen, sondern auch über weniger Angst und Depression sowie über eine verbesserte Schlafqualität (Elkins et al. 2008). Die Evidenz für die Wirksamkeit von KM-Präparaten oder Methoden bei allgemeinen Erschöpfungssymptomen ist derzeit noch gering (Sood et al. 2007); lediglich klassische Massage scheint diesbezüglich eine Verbesserung zu bringen (Listing et al. 2009).

Daneben sprechen einige Untersuchungen $\mathrm{zu}$ anderen Krebsarten als Brust- und Ovarialkrebs dafür, die gesundheitsbezogene Lebensqualität durch Bewegungsübungen zu verbessern (Mishra et al. 2012), die Stimmung zu verbessern und den erlebten Stress zu senken durch Achtsamkeitsmeditation (Musial et al. 2011), zumindest kurzfristig das Wohlgefühl durch Aromatherapie oder Massage zu verbessern (Fellowes et al. 2004), durch spirituelle Verfahren dem eigenen Bedürfnis nach Sinnerfahrung bei einer Krebserkrankung entgegenzukommen (Crammer et al. 2011), durch chinesische Kräutertees Krebs-bedingte Schmerzen kurzfristig zu mildern (Xu et al. 2007) oder andere Verfahren zu erproben, die sich bei der Behandlung lokaler Symptome Dermatitis, Xerostomie, orale Mukositis u.ä.) infolge einer Radio- oder Chemotherapie bewährt haben (im Überblick Thomas et al. 2010).

Nach der o.e. Studie von Navo et al. (2004) sprechen nur etwa die Hälfte der Frauen über den (parallelen) KM-Gebrauch mit ihrem behandelnden Arzt, größtenteils deshalb, weil sie nicht danach gefragt werden. Ihre Bereitschaft, darüber zu sprechen, ist größer, wenn sie diesen Gebrauch als sozial akzeptiert ansehen, am ehesten bei Naturheilkundlern, danach bei Homöopathen, Akupunkteuren und in diesen Berufen relativ am wenigsten bei Chiropraktikern (Saxe et al. 2008). Nach Auskunft der Frauen werden Gespräche mit ihren jeweiligen Behandlern häufig belastet durch mehr oder weniger zum Ausdruck kommende Vorwürfe, selbst am Krebs schuld zu sein oder durch unklare Aussagen über Ätiologie, Heilungschancen und das weitere Verfahren (Sered \& Agigian 2008). Aber auch für Ärzte werden Patientinnen häufig als „schwierig“ erlebt, die ihr Leiden nicht in wissenschaftlichen Kategorien klassifizieren (Hoefert \& Uehleke 2013). Andererseits könnte das Gespräch insbesondere über parallel zur einer konventionellen medikamentösen Therapie genutzte biologische KM-Verfahren auch deshalb 
nützlich sein, um möglichen Wechselwirkungen zwischen Medikamenten und Phytopharmaka vorzubeugen (im allgemeineren Zusammenhang mit Krebserkrankungen Davis et al. 2012) bzw. Verständnis zu entwickeln für Krankheitsvorstellungen der Patientinnen, die durch bestimmte KM-Philosophien beeinflusst werden (im Überblick Hoefert \& Walach 2013). Dafür spricht auch der Befund, nach dem KM-Nutzerinnen offenbar psychisch vulnerabler und empfindlicher für Stress sind als Nicht-Nutzerinnen; KM trägt bei ihnen zu einer kurzfristigen psychischen Entlastung, nicht aber zu einer Erhöhung der gesundheitsbezogenen Lebensqualität bei (Beatty et al. 2012).

\subsection{Typische gynäkologische Indikationen}

Im Folgenden werden einige typische Indikationen in der Gynäkologie aufgegriffen, bei denen Frauen häufig KM-Methoden und -Produkte wählen, wobei diese Wahl aus Überzeugung oder wegen bestimmter Medikamenten-Unverträglichkeiten erfolgen kann (s. Tab. 10). Vor allem jüngere Frauen und solche, die einen erhöhten Leidensdruck haben, neigen zum zusätzlichen Gebrauch von KM, wenn konventionelle Mittel nicht den gewünschten Erfolg bringen (vgl. Nyiriesy et al. 2011).

Tab. 10 Gynäkologische Indikationen

\begin{tabular}{|c|c|c|}
\hline Indikation & Ergebnis in Kurzform & Quelle \\
\hline \multirow[t]{5}{*}{$\begin{array}{l}\text { Prämenstruelles } \\
\text { Syndrom }\end{array}$} & $\begin{array}{l}\text { begrenzte Evidenz für Calcium, Vitamin B1 und Vit- } \\
\text { amin B6-Ergänzung }\end{array}$ & Biggs \& Demuth 2011 \\
\hline & $\begin{array}{l}\text { möglicher Nutzen für Ginkgo, Magnesium, Safran, } \\
\text { Johanniskraut, Soja und Vitamin E, keine Evidenz für } \\
\text { Magnesiumoxid und Nachtkerzenöl }\end{array}$ & Whelan et al. 2009 \\
\hline & $\begin{array}{l}\text { Bewährung von Mönchspfeffer und Narbenschenkel, } \\
\text { keine Evidenz für Johanniskraut }\end{array}$ & Dante \& Facchinetti 2011 \\
\hline & $\begin{array}{l}\text { Akupunktur bei einigen Pat. hilfreich,jedoch nicht } \\
\text { generalisierbar }\end{array}$ & Cho \& Kim 2010 \\
\hline & geringe Evidenz für Akupunktur & Kim et al. 2011 \\
\hline \multirow[t]{3}{*}{ Dysmenorrhoe } & $\begin{array}{l}\text { einige Evidenz für Vitamin B1 und Magnesium, jedoch } \\
\text { nicht für Kräuter und andere Zusatzstoffe }\end{array}$ & Proctor \& Murphy 2009 \\
\hline & $\begin{array}{l}\text { vorbehaltlich besserer Studien ist die Evidenz für } \\
\text { chinesische Kräuter vielversprechend }\end{array}$ & Zhu et al. 2008 \\
\hline & $\begin{array}{l}\text { einige Evidenz für Hitzebehandlung, Thiamine, Ma- } \\
\text { gnesium und Vitamin E }\end{array}$ & Proctor \& Farquar 2006 \\
\hline
\end{tabular}




\begin{tabular}{llll}
\hline Indikation & Ergebnis in Kurzform & Quelle \\
\hline $\begin{array}{l}\text { Evidenz für Akupunktur noch unzureichend, aber } \\
\text { vielversprechend }\end{array}$ & Cho \& Hwang 2010a \\
\hline $\begin{array}{l}\text { mögliche Wirkung von Akupunktur bei der Schmerz- } \\
\text { linderung }\end{array}$ & Smith et al. 2011d \\
\hline $\begin{array}{l}\text { deutlicher Effekt von Akupressur, insbesondere } \\
\text { aurikulare Akupressur }\end{array}$ & Cho \& Hwang 2010b \\
\hline Endometriose & $\begin{array}{l}\text { Schmerzerleichterung durch chinesische Kräuter } \\
\text { postoperativ }\end{array}$ & Wang et al. 2009 \\
\hline $\begin{array}{l}\text { Chronische } \\
\text { Vaginitis }\end{array}$ & $\begin{array}{l}\text { begrenzte Evidenz für Akupunktur } 2009 \\
\text { begrenzte Evidenz für KM generell }\end{array}$ & Zhu et al. 2011 \\
\hline
\end{tabular}

Eine weitere typische Indikation ist das chronische Beckenschmerz-Syndrom. dessen Ursachen allerdings so unterschiedlich (postoperative Komplikationen, entzündliche Prozesse, sexuelle Probleme usw., vgl. Herbert 2010) sein können, dass für KM-Interventionen nur ein begrenzter Spielraum bleibt. So glauben z.B. Sesti et al. (2010) nur eine langfristige Diättherapie mit Antioxidantien empfehlen zu können, welche die Immunfunktion verbessern helfen soll. Teilweise werden auch Interventionen wie Akupunktur, Elektrostimulation oder Palmensamen empfohlen, wie sie bei (männlicher) chronischer Prostatitis zum Einsatz kommen, um eine chirurgische Intervention zu vermeiden (vgl. Posadzki et al. 2012 zur Akupunktur, Carinci et al. 2013 zu weiteren Verfahren). Vercellini et al. (2009) weisen jedoch darauf hin, dass nur in $70-80 \%$ der Fälle eine Schmerzreduktion mit irgendeiner Methode erreichbar ist.

\subsection{Bewährung von KM in der Gynäkologie}

Es gibt weder ein Mittel noch eine Methode in der KM, das bzw. die generell für alle Indikationen empfohlen werden könnte. Vielmehr haben sich einzelne Mittel oder Methoden gegebenenfalls in sehr spezifischer Weise bewährt. So ist beispielsweise die Anwendung von Akupunktur bei durch Chemotherapie bewirkter Übelkeit durchaus erfolgreich (Kang et al. 2011), während ihre Effektivität in der Menopause oder bei Dysmenorrhoe fraglich bleibt (Smith \& Carmady 2010). Von 24 systematischen Reviews zur Akupunktur (Stand 2011), gelangen neun zu überwiegend positiven Gesamtaussagen (Ernst et al. 2011), sodass niedergelassene Gynäkologen ebenso wie Ärzte im Krankenhaus nach wie vor auf die eigene Erfahrung und auf das Experiment angewiesen bleiben. Allerdings häufen sich die empirisch ermittelten Aussagen in dem Maße, in dem KM-Forschung betrieben wird. Neben den oben genannten Ergebnissen soll auch die folgende Übersicht 
aus dem Jahr 2009 (Loyd \& Hornby 2009) eine stichwortartige Zusammenfassung der daraus ableitbaren Empfehlungen geben (s. Tab. 11).

Tab. 11 Zusammengefasste Empfehlungen (nach Lloyd \& Hornsby 2009)

\begin{tabular}{ll}
\hline Indikation & Empfehlung \\
\hline Fertilität & $\begin{array}{l}\text { Vitamin C (insbesondere vor oder während einer IVF) } \\
\text { Mönchspfeffer }\end{array}$ \\
\hline Schwangerschaft & $\begin{array}{l}\text { Effektivität von Vitamin B6 fraglich } \\
\text { Ingwer in kleinen Dosen und kurzzeitig geeignet }\end{array}$ \\
\hline Menopause & $\begin{array}{l}\text { Phytoöstrogene nicht empfehlenswert } \\
\text { Soja-haltige Produkte empfehlenswert } \\
\text { Effektivität von Traubensilberkerze fraglich }\end{array}$ \\
\hline Effektivität von Calcium und Vitamin B6 fraglich \\
Datenlage zu Magnesium, Mönchspfeffer und Nachtkerze unzureichend für \\
Empfehlungen \\
\hline kurzfristige Einnahme von Vitamin B1 empfehlenswert \\
Datenlage zu Magnesium und Vitamin E unzureichend für Empfehlungen \\
Omega-3-Fettsäuren empfehlenswert
\end{tabular}

Abschließend darf noch einmal daran erinnert werden, dass bei allen von den Patientinnen nachgefragten bzw. von diesen akzeptierten Mitteln und Methoden der KM der Grundsatz gilt, dass die persönliche Überzeugung von der Wirksamkeit gegebenenfalls höher zu gewichten ist als die vielleicht noch unzureichende wissenschaftliche Datenlage, solange nicht erwiesenermaßen eine mögliche Schädigung oder eine schädliche Dosierung dagegen spricht.

\section{Zusammenfassung}

Dieser Beitrag fasst die wichtigsten empirischen Ergebnisse zur Anwendung von Mitteln und Methoden der komplementär-alternativen Medizin in der Gynäkologie zusammen. Nach einem Überblick über die Nutzungshäufigkeiten und Nutzungsmotive thematisiert er die verschiedenen Anwendungsmöglichkeiten in den Themenbereichen Sexualität und Fertilität, Schwangerschaft und Geburt, Menopause sowie bei Krebserkrankungen und bei typischen Indikationen in der Gynäkologie. KM-Mittel und -Methoden erweisen sich nicht universell, sondern nur partiell bei bestimmten Indikationen nützlich. In der ärztlichen Beratung sollte neben der wissenschaftlichen Evidenz auch die persönliche Überzeugung der Patientin von der Wirksamkeit berücksichtigt werden. 


\section{Literatur}

Adams, I., Lui, C.W., Sibbritt, D., Broom, A., Wardle, J., Homer, C., Beck, S. (2009). Women's use of complementary and alternative medicine during pregnancy: a critical review of the literature. Birth, 36(3), 237-245.

Barragan Loayza, I.M., Sola, I., Juando Prats, C. (2011). Biofeedback for pain management during labour. Cochrane Database Syst Rev, 6, CD006168. ${ }^{11}$

Beatty, L.J., Adams, I., Sibbritt, D., Wade, T.D. (2012). Evaluating the impact of cancer on complementary and alternative medicine use, distress and health related QoL among Australian women: a prospective longitudinal investigation. Complement Ther Med, 20(1-2), 61-69.

Biggs, W.S., Demuth, R.H. (2011). Premenstrual syndrome and premenstrual dysphoric disorder. Am Fam Phys, 84(8), 918-924.

Boivin, I., Schmidt, L. (2009). Use of complementary and alternative medicines associated with a $30 \%$ lower ongoing pregnancy/live birth rate during 12 months of fertility treatment. Hum Reprod, 24(7), 1626-1631.

Borelli, F., Ernst, E. (2008). Black cocosh (Cimifuga racemosa) for menopausal symptoms: a systematic review of its efficacy. Pharmacol Res, 58(1), 8-14.

Brotto, L.A., Krychman, M., Jacobson, P. (2008). Eastern approaches for enhancing women's sexuality: Mindfulness, acupuncture, and yoga. I Sex Med, 5, 2741-2748.

Brotto, L.A., Mehak, L., Kit, C. (2009). Yoga and sexual functioning: A review. I Sex Marital Ther, 35, 378-390.

Carinci, A.J., Pathak, R., Young, M., Christo, P.J. (2013). Complementary and alternative treatments for chronic pelvic pain. Curr Pain Headache Rep, 17(2): 316.

Carpenter, C.L., Ganz, P.A., Bernstein, L. (2009). Complementary and alternative therapies among very long-term breat cancer survivors. Breast Cancer Res Treat, 116(2), 387-396.

Cheema, D., Coomarasamy, A., El Toukhy, T. (2007). Non-hormonal therapy of post-menopausal vasomotor symptoms: a structured evidence-based review. Arch Gynecol, 276(5), 463-469.

Cheong, Y.C., Hung Yu Ng, E., Ledger, W.L. (2008). Acupuncture and assisted conception. Cochrane Database Syst Rev, 4, CD006920.

Cho, S.H., Whang, W.W. (2009). Acupuncture for vasomotor menopausal symptoms: a systematic review. Menopause, 16(5), 1065-1073.

Cho, S.H., Kim, J. (2010). Efficacy of acupuncture in management of premenstrual syndrome: a systematic review. Complement Ther Med, 18(2), 104-111.

Cho, S.H., Hwang, E.W. (2010a). Acupuncture for primary dysmenorrhea: a systematic review. BJOG, 117(5), 509521.

Cho, S.H., Hwang, E.W. (2010b). Acupressure for primary dysmenorrhea: a systematic review. Compement Ther Med, 18(1), 49-56.

Clark, N.A., Will, M.A., Moravek, M.B., Fisseha, S. (2013a). A systematic review of the evidence for complementary and alternative medicine in infertility. Int I Gynaecol Obstet, 122(3), 202-206.

Clark, N.A., Will, M.A., Moravek, M.B., Fisseha, S. (2013b). Physician and patient use of and attritudes toward complementary and alternative medicine in the treatment of infertility. Int I Gynaecol Obstet, 122(3), 253257.

Cramer, H., Lauche, R., Langhorst, J., Dobos, G. (2012). Effectiveness of yoga for menopausal symptoms: a systematic review and meta-analysis of randomized controlled trials. Evid Based Complement Alternat Med, ID 863905.

Crammer, C., Kaw, C., Gansler, T., Stein, K.D. (2011). Cancer survivors' spiritual well-being and use of complementary methods: a report from the American Cancer Society's Studies of Cancer Survivors. I Relig Health, 50(1), 92-107.

Curtis, K., Weinrib, A., Katz, J. (2012). Systematic review of yoga for pregnant women: Current status and future directions. Evid Base Complement Altern Med, ID 715942.

Daley, A., Stikes-Lampard, H., MacArthur, C. (2011). Exercise for vasomotor menopausal symptoms (Review). CochraneDatabase Syst Rev, 5, CD006108.

Dante, G., Facchinetti, F. (2011). Herbal treatments for alleviating premenstrual symptoms: a systematic review. I Psychosom Obstet Gynaecol, 32(1), 42-51.

\footnotetext{
${ }^{11}$ Bei allen Cochrane Reviews wird hier immer die letzte online-verfügbare Fassung genannt.
} 
Davis, E.L., Oh, B., Butow, P.N., Mullan, B.A., Clarke, S. (2012). Cancer patient disclosure and patient-doctorcommunication of complementary and alternative medicine use: A systematic review. Oncologist, 17(11), 1475-1481.

De-Regil, L.M., Fernandez-Gaxiola, A.C., Dowswell, T., Pena-Rosas, I.P. (2010). Effects and safety of periconceptional folate supplementation for preventing birth defects. Cochrane Database Syst Rev, 10, CD007950.

De-Regil, L.M., Palacios, C., Ansary, A., Kulier, R., Pena-Rosas, I.P. (2012). Vitamin D supplementation for women during pregnancy. Cochrane Database Syst Rev, 2, CD008873.

Dhikav, V., Karmarkar, G., Gupta, R., Verma, M., Gupta, R., Gupta, S., Anand, K.S. (2010). Yoga in female sexual functions. I Sex Med, 7(Pt 2), 964-970.

Dodin, S., Blanchet. C., Marc, I., Ernst, E., Wu, T., Vaillancourt, C., Paquette, I. (2013). Acupuncture for menopausal hot flashes. Cochrane Database Syst Rev, 7, CD007410.

Downey, L., Tyree, P.T., Lafferty, W.E. (2009). Preventive screening of women who use complementary and alternative medicine providers. I Womens Health, 18(8), 1133-1143.

Dowswell, T., Bedwell, C., Lavender, T., Neilson, I.P. (2011). TENS (transcutaneous nerve stimulation) for pain relief in labour. Cochrane Database Syst Rev, 2, CD007214.

Doyle, L.W., Crowther, C.A., Middleton, P., Marret, S., Rouse, D. (2009). Magnesium sulphate for women at risk of preterm birth for neuroprotection of the fetus. Cochrane Database Syst Rev, 1, CD004661.

Duley, L., Henderson-Smart, D.J. (2009). Reduced salt intake compared to normal dietary salt, or high intake, in pregnancy. Cochrane Database Syst Rev, 3, CD001687.

Elkins, G., Marcus, I., Stearns, V., Perfect, Rajab, M.H., Ruud, C. (2008). Randomized trial of a hypnosis intervention for treatment of hot flashes among breast cancer survivors. I Clin Oncol, 26(31), 5022-5026.

El-Toukhy, T., Sunkara, S.K., Khairy, M., Dyer, R., Khalaf, Y., Coomarasamy, A. (2008). A systematic review and meta-analysis of acupuncture in in vitro fertilisation. Int I Obstet Gynaecol, 113, 1203-1213.

Ernst, E., Watson, L.K. (2011). Midwives' use of complementary/alternative treatments. Midwifery, 28(6), 772777.

Ernst, E., Posadzki, P., Lee, M.S. (2011). Complementary and alternative medicine (CAM) for sexual dysfunction and erectile dysfunction in older men and women: an overview of systematic reviews. Maturitas, 70(1), 3741.

Ernst, E., Lee, M.S., Choi, T.-Y. (2011). Acupuncture in obstetrics and gynecology: An overview of systematic reviews. Am I Chin Med, 39(3), 423-431.

Fasching, P.A., Thiel, F., Nicolaisen-Murmann, K., Rauh, C., Engel, I., Lux, M.P., Beckmann, M.W., Bani, M.R. (2007). Association of complementary methods with quality of life and life satisfaction in patients with gynecologic and breast malignancies. Support Care Cancer, 15(11), 1277-1284.

Fellowes, D., Barnes, K., Wilkinson, S. (2004). Aromatherapy and massage for symptom relief in patients with cancer. Cochrane Database Syst Rev, 2, CD002287.

Field, T. (2008). Pregnancy and labor alternative therapy research. Altern Ther Health Med, 14(5), 28-34.

Flower, A., Liu, J.P., Chen, S., Lewith, G., Little, P. (2009). Chinese herbal medicine for endometriosis. Cochrane Database Syst Rev, 3, CD006568.

Furlow, M.L., Patel, D.A., Sen, A., Liu, I.R. (2008). Physician and patient attitudes towards complementary and alternative medicine in obstetrics and gynecolocy. BMC Complement Altern Med, 8:35.

Haider, B.A., Bhutta, Z.A. (2012). Multiple-micronutrient supplementation for women during pregnancy. Cochrane Database Syst Rev, 11, CD004905.

Hajiamini, Z., Massoud, S.N., Ebadi, A., Mahboubh, A., Matin, A.A. (2012). Comparing the effects of ice massage and acupressure on labor pain reduction. Complement Ther Clin Pract, 18(3), 169-172.

Hall, E., Frey, B.N., Soares, C.N. (2011). Non-hormonal treatment strategies for vasomotor symptoms: a critical review. Drugs, 71(3), 287-304.

Hall, H.G., Griffiths, D.L., McKenna, L.G. (2011). The use of complementary and alternative medicine by pregnant women: a literature review. Midwifery, 27(6), 817-824.

Hall, H.G., McKenna, L.G., Griffiths, D.L. (2012). Midwives' support for Complementary and Alternative Medicine: a literature review. Women Birth, 25(1), 4-12.

Hall, H.G., McKenna, L.G., Griffiths, D.L. (2012). Complementary and alternative medine for induction of labour. Women Birth, 25(3), 142-148.

Han, S., Crowther, C.A., Moore, V. (2010). Gice therapy to women to prevent preterm birth after stopping threatened preterm labour. Cochrane Database Syst Rev, 7, CD000940. 
Harrigan, J.T. (2011). Patient disclosure of the use of complementary and alternative medicine to their obstetrician/gynaecologist. J Obstet Gynaecol, 31(1), 59-61.

Herbert, B. (2010). Chronic pelvic pain. Altern Ther Health Med, 16(1), 28-33.

Hodnett, E.D., Downe, S., Walsh, D. (2012). Alternative versus conventional institutional settings for birth. Cochrane Database Syst Rev, 8, CD000012.

Hoefert, H.-W., Uehleke, B. (2013). Schwierige Patientinnen in der Gynäkologie. In: Hoefert, H.-W., Härter, M. (Hrsg.). Schwierige Patienten. Bern (Huber), 129-148.

Hoefert, H.-W., Walach, H. (2013). Krankheitsvorstellungen und Motivationen bei Nutzern der komplementäralternativen Medizin. In: Hoefert, H.-W., Brähler, E. (Hrsg.). Krankheitsvorstellungen von Patienten - Herausforderung für Medizin und Psychotherapie. Lengerich (Pabst), 279-306.

Huntley, A.L., Ernst, E. (2003). A systematic review of herbal medicinal products for the treatment of menopausal symptoms. Menopause, 10(5), 465-476.

Innes, K.E., Selfe, T.K., Vishnu, A. (2010). Mind-body therapies for menopausal symptoms: A systematic review. Maturitas, 66(2), 135-149.

Jaganfar, S., Jaafar, S.H. (2012). Effects of restricted caffeine intake by mother on fetal, neinatal and pregnancy outcome. Cochrane Database Syst Rev, 2, CD006965.

Kalder, M., Knoblauch, K., Hrgovic, I., Münstedt, K. (2011). Use of complementary and alternative medicine during pregnancy and delivery. Arch Gynecol Obstet, 283(3), 475-482.

Kang, H.S., Jeong, D., Kim, D.I., Lee, M.S. (2011). The use of acupuncture for managing gynaecologic conditions: An overview of systematic reviews. Maturitas, 68(4), 346-354.

Keenan, N.L., Saralyn, M., Fugh-Berman, A., Browne, D., Kaczmarczyk, J., Hunter, C. (2003). Severity of menopausal symptoms and use of both concentional and complementary/alternative therapies. Menopause, 10(6), 507-515.

Khianman, B., Pattanittum, P., Thinkhamrop, J., Lumbiganon, P. (2012). Relaxation therapy for preventing and treating preterm labour. Cochrane Database Syst Rev, 8, CD007426.

Kim, S.Y., Park, H.J., Lee, H., Lee, H. (2011). Acupuncture for premenstrual syndrome: a systematic review and meta-analysis of randomised controlled trials. BJOG, 118(8), 899-915.

Laakmann, E., Grajecki, D., Doege, K., zu Eulenburg, C., Buhling, K.J. (2012). Efficacy of Cimifuga racemosa, Hypericum perforatum and Agnus castus in the treatment of climacteric complaints: a systematic review. Gynecol Endocrinol, 28(9), 703-709.

Leach, M.J., Moore, V. (2012). Black cocosh (Cimifuga spp.) for menopausal symptoms. Cochrane Database Syst Rev, 9, CD007244.

Lee, M.S., Kim, J.I., Ha, J.Y., Boddy, K., Ernst, E. (2009). Yoga for menopausal symptoms: a systematic review. Menopause, 16(3), 602-608.

Lethaby, A.E., Brown, I., Marjoribanks, I., Kronenberg, F., Roberts, H., Eden, I. (2007). Phytoestrogens for vasomotor menopausal symptoms. Cochrane Datebase Syst Rev, 4, CD001395.

Li, L., Dou, L., Leung, P.C., Wang, C.C. (2012). Chinese herbal medicines for threatened miscarriage. Cochrane Database Syst Rev, 5, CD008510.

Licciardone, J.C., Buchanan, S., Hensel, K., Ling, H.H., Fulda, K.G., Stoll, S.T. (2010). Osteopathic manipulative treatment of back pain and related symptoms during pregnancy: A randomized controlled trial. Am J Obstet Gynecol, 202(1), 43e1-43e8.

Lim, C.E., Wilkinson, J.M., Wong, W.S., Cheng, N.C. (2009). Effect of acupuncture on induction of labor. I Altern Complement Med, 15(11), 1209-1214.

Lindenmeyer, A., Jamie, K., Griffiths, F., LeGaré, F. (2011). „They're made in factories and not by witches on the alotment": A qualitative study of midlife women in the United Kingdom, exploring their approaches to complementary and alternative medicines. Health Care Women Int, 32, 1046-1067.

Listing, M., Reisshauer, A., Krohn, M., Voigt, B., Tjahono, G., Becker, J., Klapp, B.F., Rauchfuss, M. (2009). Massage therapy reduces physical discomfort and improves mood disturbances in women with breast cancer. Psychooncology, 18(12), 1290-1299.

Lloyd, K.B., Hornsby, L.B. (2009). Complementary and alternative medications for women's health issues. Nutr Clin Pract, 24(5), 589-608.

Louik, C., Gardiner, P., Kelley, K., Mitchell, A.A. (2010). Use of herbal treatments in pregnancy. Am I Obstet Gynecol, 202(5), 439e1-439e10.

Lunny, C.A., Fraser, S.N. (2010). The use of complementary and alternative medicines among a sample of Canadian menopausal-aged women. J Midwifery Womens Health, 55(4), 335-343. 
Ma, H., Sullivan-Halley, Smith, A.W., Neuhouser, M.I., Alfano, C.M., Meeske, K., George, S.M., McTiernan, A., McKean-Cowdin, R., Baumgartner, K.B., Ballard-Barbash, R., Bernstein, L. (2011). Estrogenic botanical supplements, health-related quality of life, fatigue, and hormone-related symptoms in breast cancer survivors: a HEAL study report. BMC Complement Altern Med, 11:109.

Madaschi, C., Braga, D.P., Fuguera C., laconelli, A., Borges, E. (2010). Effect of acupuncture on assisted reproduction treatment outcomes. Acupunct Med, 28(4), 180-184.

Madden, K., Middleton, P., Cyna, A.M., Matthewson, M., Jones, L. (2012). Hypnosis for pain management during labour and childbirth. Cochrane Database Syst Rev, 11, CD009356.

Marc, I., Toureche, N., Ernst, E., Hodnet, E.D., Blanchet, C., Dodin, S., Nioya, M.M. (2011). Mind-body interventions during pregnancy for preventing or treating women's anxiety. Cochrane Database Syst Rev, 7, CD007559.

Matthews, A., Dowswell, T., Haas, D.M., Doyle, M., O'Mathuna, D.P. (2010). Interventions for nausea and vomiting in early pregnancy. Cochrane Database Syst Rev, 9, CD007575.

Mazaro-Costa, R., Andersen, M.L., Hachul, H., Tufik, S. (2010). Medicinal plants as alternative treatments for female sexual dysfunction: utopian vision or possible treatment in climacteric women? I Sex Med, 7(11), $3695-3714$.

Mishra, S.I., Scherer, R.W., Geigle, P.M., Berlanstein, D.R., Topaloglu, O., Gotay, C.C., Snyder, C. (2012). Exercise interventions on health-related quality of life for cancer survivors (review). Cochrane Database Syst Rev, 8 , CD007566.

Mitchell, M. (2010). Risk, pregnancy and complementary and alternative medicine. Complement Ther Clin Pract, 16(2), 109-113.

Mori, R., Ota, E., Middleton, P., Tobe-Gai, R., Mahomed, K., Bhutta, Z.A. (2012). Zinc supplementation for improving pregnancy and infant outcome. Cochrane Database Syst Rev, 7, CD000230.

Morrow, P.K.H., Mattair, D.N., Hortobagyi, G.N. (2011). Hot flashes: A review of pathophysiology and treatment modalities. Oncologist, 16, 1658-1664.

Moussally, K., Berard, A. (2012). Exposure to specific herbal products during pregnancy and the risk of low birth weight. Altern Ther Health Med, 18(2), 36-43.

Mueller, C.M., Mai, P.I., Bucher, J., Peters, J.A., Loud, I.T., Greene, M.H. (2008). Complementary and alternative medicine use among women at increased genetic risk of breast and ovarian cancer. BMC Complement Altern Med, 8:17.

Münstedt, K., Schroter, C., Brüggmann, D., Tinneberg, H.R., von Georgi, R. (2009a). Use of complementary and alternative medicine in departments of obstetrics in Germany. Forsch Komplementmed, 16(2), 111-116.

Münstedt, K., Brenken, A., Kalder, M. (2009b). Clinical indications and perceived effectiveness of complementary and alternative medicine in departments of obstetrics in Germany: a questionnaire study. Eur / Obstet Gynecol Reprod Biol, 146(1), 50-54.

Münstedt, K., Thienel, J., Hrogovic, I., Hackethal, A., Kalder, M., Misselwitz, B. (2011). Use of acupuncture and other CAM methods in obstetrics: an analysis of 409.413 deliveries from Hesse, Germany. J Altern Complement Med, 17(5), 421-426.

Münstedt, K., Dütemeyer, V., Hübner, I. (2013). Patients' considerations behind the use of methods from complementary and alternative medicine in the field of obstetrics in Germany. Arch Gynecol Obstet, 288(3), 527530.

Musial, F., Büssing, A., Heusser, P., Choi, K.E., Ostermann, T. (2011). Mindfulness-based stress reduction for integrative cancer care: a summary of evidence. Forsch Komplementmed, 18(4), 192-202.

Navo, M.A., Phan, I., Vaughan, C., Palmer, L., Michaud, L., Jones, K.L., Bodurka, D.C., Basen-Wnquist, K., Hortobagyi, G.N., Kavanagh, I.J., Smith, J.A. (2004). An assessment of the utilization of complementary and alternative medication in women with gynecologic or breast malignancies. J Clin Oncol, 4(15), 671-677.

Nedrow, A., Miller, J. Walker, M., Nygren, P., Hoyt Huffman, L., Nelson, H.D. (2006). Complementary and alternative therapies for the management of menopause-related symptoms. Arch Intern Med, 166, 1453-1465.

North American Menopause Society (NAMS) (2004). Treatment of menopause-associated vasomotor symptoms: position statement of The North American Menopause Society. Menopause, 11(1), 11-33.

Nyirjesy, P., Robinson, I., Matthew, L., Lev-Sagie, A., Reyes, I., Culhane, I.F. (2011). Alternative therapies in women with chronic vaginitis. Obstet Gynecol, 117(4), 856-861.

Othman, M., Alfirevic, Z., Neilson, I.P. (2012). Probiotics for preventing preterm labour. Cochrane Database Syst Rev, 1, CD005941.

Pachman, D.R., Jones, J.M., Loprinzi, C.L. (2010). Management of menopause-associated vasomotor symptoms: Current treatment options, challenges and future directions. Int I Women's Health, 2, 123-135. 
Pena-Rosas, I.P., De-REgil, L.M., Dowswell, T., Viteri, F.E. (2012). Intermittant oral iron supplementation during pregnancy. Cochrane Database Syst Rev, 7, CD009997.

Pennick, V.E., Young, G. (2007). Interventions for preventing and trating pelvic and back pain in pregnancy. 2, Cochrane Database Syst Rev. CD001139.

Pitkin, J. (2012). Alternative and complementary therapies for the menopause. Menopause Int, 18, 20-27.

Posadzki, P., Zhang, I., Lee, M.S., Ernst, E. (2012). Acupuncture for chronic nonbacterial prostatitis/chronic pelvic pain syndrome: A systematic review. I Androl, 33(1), 15-21.

Proctor, M., Farquar, C. (2006). Diagnosis and management of dysmenorrhea. BMI, 332, 1134-1138.

Proctor, M., Murphy, P.A. (2009). Vitamin B1 and magnesium may both help reduce the pain of dysmenorrhea. Cochrane Database Syst Rev, 2, CD002124.

Qu, H.B., Dengfeng, W., Wu, T., Marjoribanks, J., Ying, S., Haijun, J., Zhang, J., Hu, L. (2011). Chinese herbal medicine in the treatment of ectopic pregnancy. Cochrane Database Syst Rev, 7, CD006224.

Rayner, J.A., Willis, K., Burgess, R. (2011). Women's use of complementary and alternative medicine for fertility enhancement: a review oft he literature. J Altern Complement Med, 17(8), 685-690.

Rhode, J.M., Patel, D.A., Sen, A., Schimp, V.L., Johnston, C.M., Liu, J.R. (2008). Perception and use of complementary and alternative medicine among gynecologic oncology care providers. Int I Gynaecol Obstet, 103(2), 111-115.

Ried, K., Stuart, K. (2011). Efficacy of Traditional Herbal Medicine in the management of female infertility: a systematic review. Complement Ther Med, 19(6), 319-331.

Roberts, H. (2010). Safety of herbal medicinal products in women with breast cancer. Maturitas, 66(4), 363-369.

Rumbold, A., Crowther, C.A., Haslam, R.R., Dekker, G.A., Robinson, I.S., ACTS Study Group (2006). Vitamins C and E and the risks of preeclampsia and perinatal complications. N Engl I Med, 354(17), 1796-1806.

Rumbold, A., Middleton, P., Pan, N., Crowther, C.A. (2011). Vitamin supplementation for preventing miscarriage. Cochrane Database Syst Rev, 1, CD004073.

Sado, M., Ota, E., Stickley, A., Mori, R. (2012). Hypnosis during pregnancy, childbirth, and the postnatal period for preventing postnatal depression. Cochrane Database Syst Rev, 6, CD009062.

Saquib, I., Madlensky, L., Kealey, S., Saquib, N., Natarajan, L., Newman, V.A., Patterson, R.E., Pierce, J.P. (2011). Classification of CAM use and its correlates in patients with early-stage breast cancer. Integr Cancer Ther, 10(2), 138-147.

Saxe, G.A., Madlensky, L., Lealey, S., Wu, D.P., Freeman, K.L., Pierce, I.P. (2008). Disclosure to physicians of CAM use by breast cancer patients: Findings from the Women's healthy Eating and Living Study. Integr Cancer Ther, 7(3), 122-129.

Say, L., Gülmezoglu, A.M., Hofmeyr, G.J. (2010). Maternal nutrient supplementation for suspected impaired fetal growth. Cochrane Database Syst Rev, 1, CD000148.

Schaffir, I., McGee, A., Kennard, E. (2009). Use of nonmedical treatments by ifertility patients. I Reprod Med, 54(7), 415-420.

Sered, S., Agigian, A. (2008). Holistic sickening: breast cancer and the discursive worlds of complementary and alternative practioners. Sociol Health IIln, 30(4), 616-631.

Sesti, F., Capozzolo, T., Pietropolli, A., Collalti, M., Bollea, M.R., Piccione, E. (2010). Dietary therapy: a new strategy for managementz of chronic pelvic pain. Nutr Res Rev, 1-8.

Shams, T., Setia, M.S., Hemmings, R., McCusker, I., Sewitch, M., Ciampi, A. (2010). Efficacy of black cocoshcontaining preparations on menopausal symptoms: a meta-analysis. Altern Ther Health Med, 16(1), 36-44.

Shou, C., Li, J., Liu, Z. (2011). Complementary and alternative medicine in the treatment of menopausal symptoms. Chin J Integr Med, 17(12), 883-888.

Sideras, K., Loprinzi, C.L. (2010). Nonhormonal management of hot flashes for women on risk reduction therapy. I Natl Compr Canc Netw, 8, 1171-1179.

Slavin, S.L., Rogers, R.G., Komesu, Y., Omotosho, T., Hammil, S., Lewis, C., Sapien, R. (2010). Complementary and alternative medicine (CAM) use in women with pelvic floor disorders: a cohort study. Int Urogynecol I, 21(4), 431-437.

Smith, C.A. (2010). Homoeopathy for induction of labour. Cochrane Database Syst Rev, 4, CD003399.

Smith, C.A., Cochrane, S. (2009). Does acupuncture have a place as an adjunct treatment during pregnancy? A review of randomized controlled trials and systematic reviews. Birth, 36(3), 246-253.

Smith, C.A., Carmady, B. (2010). Acupuncture to treat common reproductive health complaints: An overview of the evidence. Auton Neurosci, 157(1-2), 52-56. 
Smith, C.A., Collins, C.T., Cyna, A.M., Cowther, C.A. (2010). Complementary and alternative therapies for pain management in labour. Cochrane Database Syst Rev, 4, CD003521.

Smith, C.A., Collins, C.T., Crowther, C.A., Levett, K.M. (2011a). Acupuncture or acupressure for pain management in labour. Cochrane Database Syst Rev, 7, CD009232.

Smith, C.A., Collins, C.T., Crowther, C.A. (2011b). Aromatherapy for pain management in labour. Cochrane Database Syst Rev, 7, CD009215.

Smith, C.A., Levett, K.M., Collins, C.T., Crowther, C.A. (2011C). Relaxation techniques for pain management in labour. Cochrane Database Syst Rev, 12, CD009514.

Smith, C.A., Zhu, X., He, L., Song, I. (2011d). Acupuncture for primary dysmenorrhea. Cochrane Database Syst Rev, 1, CD007854.

Smith, C.A., Levett, K.M., Collins, C.T., Jones, L. (2012). Massage, reflexology and other manual methods for managing pain in labour. Cochrane Database Syst Rev, 2, CD009290.

Smith, J.A., Badell, M.L., Kunther, A., Palmer, J.L., Dalrymple, J.L., Ramin, S.M. (2012). Use of complementary and alternative medications among patients in an obstetrics and gynecological clinic. I Reprod Med, 57(9-10), 390-396.

Smith, J.F., Eisenberg, M.L., Millstein, S.G., Nachtigall, R.D., Shindel, A.W., Wing, H., Cedars, M., Pasch, L., Katz, P.P., Infertility Outcomes Program Project Group (2010). The use of complementary and alternative fertility treatment in couples seeking fertility care: Data from a prospective cohort in the United States. Fertil Steril, 93(7), 2169-2174.

Sood, A., Barton, D.L., Bauer, B.A., Loprinzi, C.L. (2007). A critical review of complementary therapies for cancerrelated fatigue. Integr Cancer Ther,6(1), 8-13.

Sunita, P., Pattanayak, S.P. (2011). Phytoestrogens in postmenopausal indications: A theoretical perspective. Pharmacogn Rev, 5(9), 41-47.

Thacker, H.L. (2011). Assessing risks and benefits of nonhormonal treatments for vasomotor symptoms in perimenopausal and postmenopausal women. I Womens Health, 20(7), 1007-1016.

Thaver, D., Saeed, M.A., Bhutta, Z.A. (2006). Pyridoxine (vitamin B6) supplementation in pregnancy. Cochrane Database Syst Rev, 2, CD000179.

Thomas, J., Beinhorn, C., Norton, D., Richardson, M., Sumler, S.S., Frenkel, M. (2010). Managing radiation therapy side effects with complementary medicine. I Soc Integr Oncol, 8(2), 65-80.

Tournaire, M., Theau-Yonneau, A. (2007). Complementary and alternative approaches to pain relief during labor. Evid Based Complement Alternat Med, 4(4), 409-417.

Upchurch, D.M., Chyu, L., Greendale, G.A., Utts, I., Bair, Y.A., Zhang, G., Gold, E.B. (2007). Complementary and alternative medicine usw among American women: findings from The National Health Interview Survey. I Womens Health, 16(1), 102-113.

Vercellini, P., Vigano, P., Somigliana, E., Abbiati, A., Barbara, G., Fedele, L. (2009). Medical, surgical and alternative treatments for chronic pelvic pain in women: a descriptive review. Gynecol Endocrinol, 25(4), $208-221$.

Vincent, A., Barton, D.L., Mandrekar, J.N., Cha, S.S., Zais, T., Wahner-Roedler, D.L., Keppler, M.A., Kreitzer, M.J., Loprinzi, C. (2007). Acupuncture for hot flashes: a randomized, sham-controlled clinical study. Menopause, 14(1), 45-52.

von Studnitz, F.S., Eulenburg, C., Mueck, A.O., Buhling, K.J. (2013). The value of complementary and alternative medicine in the treatment of climacteric symptoms: Results of a survey among German gynecologists. Complement Ther Med, 21(5), 492-495.

Wang, M.C., Hsu, M.C., Chien, L.W., Kao, C.H., Liu, C.F. (2009). Effects of auricular acupressure on menstrual symptoms and nitric oxide for women with primary dysmenorrhea. I Altern Complement Med, 15(3), 235242.

Warriner, S., Bryan, K., Brown, A.M. (2013). Women's attitude towards the use of complementary and alternative medicines (CAM) in pregnancy. Midwifery, pii: S0266-6138(13)00095-8

Watson, C.J., Pirotta, M., Myers, P. (2012). Use of complementary and alternative medicine in recurrent vulvovaginal candidiasis - results of a practioner survey. Complement Ther Med, 20(4), 218-221.

Weiss, D.A., Harris, C.R., Smith, J.F. (2011). The use of complementary and alternative fertility treatments. Curr Opin Obstet Gynecol, 23(3), 195-199.

Whelan, A.M., Jurgens, T.M., Naylor, H. (2009). Herbs, vitamins and minerals in the treatment of premenstrual syndrome; a systematic review. Can I Clin Pharmacol, 16(3), e407-e429.

Wong, V.C., Lim, C.E., Luo, X., Wong, W.S. (2009). Current alternative and complementary therapies used in menopause. Gynecol Endocrinol, 25(3), 166-174. 
Xu, L., Lao, L.X., Ge, A., Yu, S., Li, J., Mansky, P.J. (2007). Chinese herbal medicine for cancer pain. Integr Cancer Ther, 6(3), 208-234.

Xu, L.W., Jia, M., Salchow, R., Kentsch, M., Cui, X.J., Deng, H.Y., Sun, Z.J., Kluwe, L. (2012). Efficacy and side effects of chinese herbal medicine for menopausal symptoms: A critical review. Evid Based Complement Alternat Med, ID 568106.

Zhu, X., Proctor, M., Bensoussan, A., Wu, E., Smith, C.A. (2008). Chinese herbal medicine for primary dysmenorrhoea. Cochrane Database Syst Rev, 2, CD005288.

Zhu, X., Hamilton, K.D., McNicol, E.D. (2011). Acupuncture for pain in endometriosis. Cochrane Database Syst Rev, 9, CD007864. 